\title{
CT and MRI Imaging Features and Grades of Liver Iron Deposition
}

\author{
Qianwen Huang, Simin Lin, Shaoyin Duan a \\ Zhongshan Hospital of Xiamen University, 209 South Hubin Rd., Xiamen 361004, China
}

\begin{abstract}
CT and MRI imaging features and grades of liver iron deposition (LID) were discussed and evaluated in clinical application. The imaging and laboratory data of 20 patients with LID were analyzed retrospectively. CT value of liver were measured and MR signal intensity ratio of muscle/liver calculated.A new imaging grade of LID were made with CT and MR, and the grade correlation was analyzed between CT, MR and SF. All of 20 patients with LID, 19 cases were $\mathrm{CT}$ examination, their $\mathrm{CT}$ value ranging of liver were from $55 \mathrm{HU}$ to $116 \mathrm{HU}$, of which more than $72 \mathrm{HU}$ were in 7 cases and $72 \mathrm{HU}$ or less in 12.14 cases were MR examination with the low signal of liver, and the inverse phase signal of T1WI was higher than that of the positive phase. There were no significant difference among the grade of CT, T1WI, T2WI and $\mathrm{SF}(\mathrm{P}>0.05)$, and there were the positive correlation of grade between CT, T1WI, T2WI and SF ( $\mathrm{rs}=0.803,0.847,0.677$ respectively), and of values between CT, T1WI SIR, T2WI SIR and $\mathrm{SF}(\mathrm{rs}=0.454,0.538,0.811$ respectively). CT can show the LID with high-density, but the mild LID can be with normal density, which is the false negative. MRI can show LID with low-signal, of which T2WI is the lower than T1WI, it is beneficial to diagnose the early LID. Grades of CT and MR have good consistent with that of SF, and can reflect the severity of LID, particularly that of MR-T1WI grading.
\end{abstract}

\section{Introduction}

Liver iron deposition (LID), also known as hepatic hemochromatosis, hepatic hemoglobinosis, or bronze diabetes [1], etc. Liver is the earliest and most serious implicated organ when the iron content is too much in the body, for it is the main organ for storing iron. Normally, iron can be transported in blood plasma by transferrin binding iron (TBI). However, the excess iron will exists with the form of non-transferrin binding iron (NTBI), when iron was overload in the body for the intrahepatic or extrahepatic diseases. Many diseases lead to LID, such as Cooley's anemia, blood transfusion dependent anemia, hematologic malignancy and various chronic liver diseases. Since there is no active iron excretion system, long-term accumulation of iron in the liver can cause and promote liver fibrosis, cirrhosis or even liver cancer, which is 200 times higher than that of the normal population. Therefore, the early diagnosis of hepatic iron deposition is very necessary. At present, the primary methods of clinical diagnosis of LID include the blood test, CT, MRI, liver biopsy, superconducting quantum interferometer (SQUID) and so on. In this study, 20 patients with LID, diagnosed in our hospital from February 2011 to July 2017, were retrospectively analyzed in order to discuss their clinical and imaging features, improve the diagnostic accuracyand evaluate the LID garding of CT and MRI.

\section{Materials and methods}

\footnotetext{
a Corresponding author: xmdsy@xmu.edu.cn
}

\subsection{Research subjects}

Collected 20 patients with LID (serum ferritin SF > $1000 \mathrm{ng} / \mathrm{ml}$ or/and pathologically confirmed) in our hospital from February 2011 to July 2017, including 13 males and 7 females, aged 4-79 years, with an average age of $43.2 \pm 25.4$ years. CT, MRI, SF examination was all performed in 13 cases, of which 2 cases were confirmed by pathology; 6 cases had only CT, SF examination; only 1 case had MRI, SF examination and was confirmed by pathology.

\subsection{Clinical data}

In all of 20 patients with LID, except for 5 patients with Severe $\beta$-thalassemia and 1 with hereditary hemochromatosis, the remaining 14 cases were found by CT and / or MR examination due to other diseases. There were 1 case of pancreatic carcinoma, 1 case of ileocecal carcinoma, 1 case of gastric antrum carcinoma, 1 case of duodenal carcinoma, 1 case of transverse colon cancer, 1 case of rectal cancer, 1 case of chronic hepatitis $B$ cirrhosis, 1 case of gallstone with cholecystitis, 1 case of esophageal carcinoma, 2 cases of liver cancer, 1 case of Chronic renal failure, 1 case of multiple myeloma, and jaundice in 1 case; There were 4 cases complicated anemia and 7 cases complicated liver diseases (hepatitis $\mathrm{B}$, cirrhosis and liver cancer). The levels of SF in 20 cases were higher than the normal value, the highest is up to $7169.0 \mathrm{ng} / \mathrm{ml}$. 


\subsection{CT value measurement of liver}

Three regions of interest (ROI) were selected in the parenchyma of the right posterior lobe of the liver, avoid the imaging artifact, intrahepatic bile ducts and the blood vessels by visible to the naked eye, measure the $\mathrm{CT}$ value 3 times and take the average value. MR signal observation: three doctors with more than 10 years' experience in MR diagnosis were selected to observe and analyze.

\subsection{MR signal measurement of liver}

The signal intensity (SI) of liver and paravertebral muscles were measured by MR on T1WI and T2WI respectively. The ROI in liver measurement was selected in the right posterior lobe of the liver (left lobe of liver should not be selected as ROI because of heart and abdominal aorta pulsation artifact), avoid piping systems and areas with artifact in order to reduce partial volume effects. The size of ROI used in the measurement of each part is the same as far as possible to reduce the impact on the accuracy of the measurement. Calculate the signal intensity ratio (SIR) of muscle / liver (M/ L), all of the data above measured 3 groups and take the average value.

\subsection{Statistical method}

Statistical analysis was performed using SPSS 13.0 package. The correlation between CT value, MR SIR and SF value was analyzed by Wilcoxon rank sum test and Spearman's correlation analysis. Quantitative comparison of SF, CT and MRI were compared by ANOVA, welch correction test and Spearman's correlation analysis. $\mathrm{P}<0.05$ was the standard of statistical difference.

\section{Results}

\subsection{CT imaging and analysis}

All of 20 patients with LID, 19 cases were CT examination, the CT value ranging of liver were from $55 \mathrm{HU}$ to $116 \mathrm{HU}$, of which more than $72 \mathrm{HU}$ were in 7 cases (Fig.1) and $72 \mathrm{HU}$ or less in 12. CT grading criteria: according to the measured $\mathrm{CT}$ value, the mild $\mathrm{CT}$ value was $\leq 72 \mathrm{HU}$, the moderate $72<\mathrm{CT}$ value $<100 \mathrm{HU}$ and the severe $\mathrm{CT}$ value $\geq 100 \mathrm{HU}$ (Fig. 1).

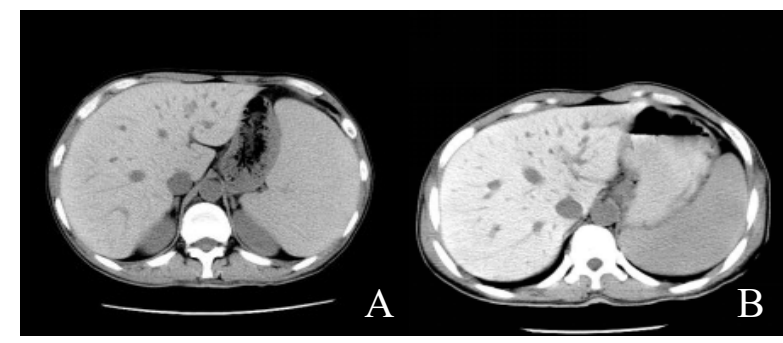

Figure 1. CT showed that the density of liver was increased, manifested as "white liver". A and B is the different cases which $\mathrm{CT}$ values were about $87 \mathrm{HU}$ and $116 \mathrm{HU}$, respectively. Simultaneous splenomegaly.

\subsection{MR imaging and analysis}

14 cases were MR examination, the low signal of liver were showed in the T1WI and T2WI images, showing below or near the same level paraspinal muscles, manifested as "black liver", and the T2WI signal is lower than T1WI. The inverse phase signal is higher than the positive phase signal on the gradient echo T1WI positive and negative phase image(Fig.2). MR grading method and criteria: Grading the severity of LID on MRI images by selecting the tissues that were not prone to iron deposition (paravertebral muscles, vertebral body and lung): mild liver signals were equal to or lower than those of paravertebral muscles on the same plane; The moderate liver signal is equal to or lower than the same plane vertebra signal; the severe liver signal is equal to or lower than the lung tissue signal.

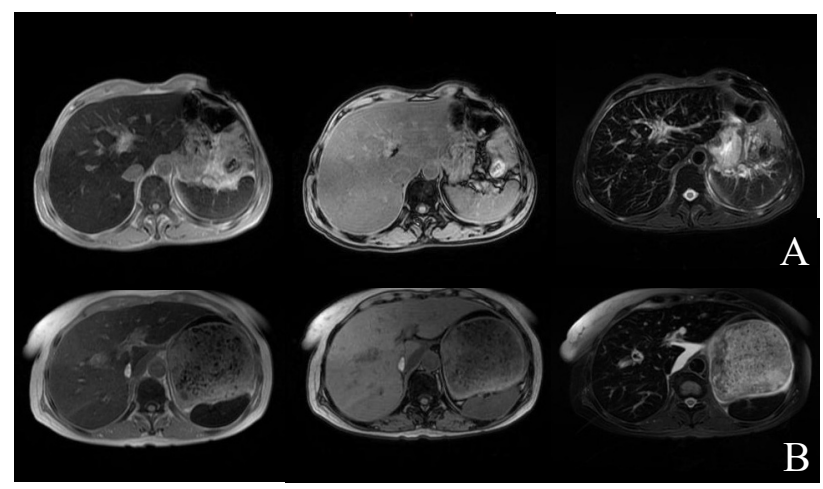

Figure 2. A was a MRI image of the same patient with T1WI positive phase, T1WI inverse phase and T2WI sequence images. Both the positive and inverse phase of T1WI and the signal of T2WI liver were decreased, showing "black liver", and the decrease of T2WI signal was more significant. At the same time, the inverse phase signal on T1WI was higher than the positive phase signal of T1WI, is contrary to fatty liver; B was another case of MRI imaging, and the signal of each sequence was the same as A.

\subsection{Data of SF CT and MRI and comparison}

There were 20 cases of SF and 19 cases of Mr in LID patients. SF, CT, MRI imaging grading of LID compared with statistics (Table 1). The results of Spearman's correlation test showed that the $\mathrm{CT}$ indexing had a strong positive correlation with $\mathrm{SF}(r=0.803, P=0.000)$; there was a very strong positive correlation between $\mathrm{T} 1$ and $\mathrm{SF}$ $(r=0.847, \quad P=0.000)$ and a strong positive correlation between T2 and SF $(r=0.677, P=0.008)$.

Table 1. LID grading and comparison )n, $\%($

\begin{tabular}{|c|c|c|c|}
\hline Grade & Mild & Moderate & Severe \\
\hline SF value $) \mathrm{n}=20($ & $7) 35.0($ & $7) 35.0($ & $6) 30.0($ \\
\hline CT value $) \mathrm{n}=19($ & $12) 63.2($ & $4) 21.0($ & $3) 15.8($ \\
\hline MR-T1 $) \mathrm{n}=14($ & $8) 57.1($ & $5) 35.7($ & $1) 7.1($ \\
\hline MR-T2 $) \mathrm{n}=14($ & $2) 14.3($ & $8) 57.1($ & $4) 28.6($ \\
\hline
\end{tabular}

Note: Wilcoxon rank sum test showed no significant difference between SF, CT, T1WI, T2WI scores $(\mathrm{P}=0.790, \mathrm{P}=0.852)$ 


\section{Discussion}

\subsection{Iron metabolism and clinical manifestations}

The normal sources of iron are mainly from food intake from animals $\left(\mathrm{Fe}^{2+}\right)$ or from plants $\left(\mathrm{Fe}^{3+}\right)$ and iron released from aging red blood cells. Iron is mainly absorbed from the duodenum and 1/4 segment of intestinal mucosa in upper jejunum. The excess iron is stored in the form of ferritin and hemosiderin in the mononuclear macrophage system of liver, spleen, bone marrow and other organs. When iron deposits slowly increase in the liver, it may damage the liver cells through oxidative stress or immune disorder, induce hepatic inflammatory lesions, hepatocyte necrosis, cirrhosis and $\mathrm{HCC}$ precancerous lesions. The onset of the disease is hidden and progress slowly, mostly in middle age, male are more than female, probably because women have menstrual onset. The typical triple signs of the disease are skin pigmentation, cirrhosis and secondary diabetes, but the incidence is only $3 \% \sim 8 \%$. No typical triple signs were found in this group and $70 \%$ of LID was found by accident when CT or MR examination of other diseases.

\subsection{Clinical classification and diagnosis}

LID can be classified into two main categories according to the etiology, including the primary iron deposition and secondary iron deposition. In this group of 20 cases, only 1 case of hereditary hemochromatosis was primary and the other 19 cases were secondary. Therefore, the diagnosis and treatment of secondary LID should be noticed and avoided misdiagnosis. The order of reliability of laboratory and imaging examination in diagnosing on LID is: HFE-DNA analysis, MRI, dual-energy CT, liver iron index, erythrocyte basic ferritin, serum ferritin and transferrin saturation (SF) [2]. The common laboratory examination are SF and TS, which combined use can improve the accuracy of evaluation. Liver biopsy is the gold standard for LID, but it has a certain risk because of it's invasive. There is only 3 cases in the group have biopsy pathological data, is not extensive in clinical application. Imaging examination was mainly $\mathrm{CT}$ and MR, there was no difference between primary and secondary iron deposition in liver. Because of noninvasive, imaging examination are more clinical application, it is one of the important methods in the clinic. In this group, 14 cases are accidental positive diagnosis of target area. HFE-DNA analysis is the final basis for the diagnosis of hereditary iron deposition and is the only indicator that can be used to distinguish hereditary from secondary[3].

\subsection{Imaging features}

CT findings of LID showed the liver with CT value of $72 \sim 132 \mathrm{HU}$ or higher. The density of hepatic parenchyma was strongly contrasted with intrahepatic blood vessels, and showed as "white liver". The CT value was positively correlated with the degree of iron deposition in the liver, which could reflect the iron content in the liver. However, when there is fatty liver, the CT value of liver will decrease because of the effect of fat, which leads to the error in judging the severity of liver iron deposition, and even false negative[4]. MR show the liver as "black liver", and the change of signal intensity was related to the degree of iron deposition[5]. All the 14 cases of MR examination showed that the signal homogeneity of liver parenchyma was decreased and T2WI is more obvious than T1WI, which was consistent with the literature reports. Combining T2 "black liver" with T1WI positive and negative phase specificity can improve the accuracy and sensitivity of LID diagnosis[6].

\subsection{Deficiencies and prospects}

SF is susceptible to active inflammation, liver disease, tumor, hemolysis and other factors. In this study, some cases were complicated with tumor, inflammation and so on. Only one laboratory index of SF was used to evaluate the degree of iron deposition in liver, which lacked some accuracy. Imaging findings of LID were graded, without a corresponding quantitative study. The quantitative methods of LID include SQUID, MR and dual-energy CT etc. Using MR technique to realize iron quantification, the sampling variability is low, and the accuracy of evaluating iron content in whole body is high, is considered the standard for the diagnosis and monitoring of iron overload diseases currently. Dual-energy CT can obtain virtual iron content (VIC) image to evaluate LIC quantification by iron specific three-substance separation algorithm, which has similar diagnostic accuracy to MR, and not affected by liver steatosis[7,8,9].

\section{References}

1. X.P. Lin, H. Wei. CT diagnostic value of hepatic hemochromatosis. Journal of Youjiang Medical University for Nationalities, 33, 504 (2011).

2. X.J. Guo, C.L. Wang, P.C. Liu, Q. Shi, X.J. Xiang, H.M. Shan. MRI diagnosis of Hemochromatosis. Journal of Medical Imaging, 20, 7 (2010).

3. R.W. Wang. Diagnosis of hemochromatosis. Journal of China Pediatric Blood and Cancer, 6,193 (2001).

4. G.D. Yang, P.X. Lu, R.X Ye. CT diagnosis of hepatic iron hypertrophy in a case of hemochromatosis. Journal of Medical Imaging, 17, 888 (2007).

5. B.J. Trottier, L.J. Burns, T.E. Defor, S. Cooley, N.S. Majhail. Association of iron overload with allogeneic hematopoietic cell transplantation outcomes: a prospective cohort study using R2-MRI-measured liver iron content.Blood, 122, 1678 (2013).

6. S.L. Yu, L.B. Ma, Y. Liu. The value of magnetic resonance imaging in the diagnosis of hepatic hemochromatosis. Chinese Journal of New Clinical Medicine, 7, 921( 2014).

7. X.F. Luo, X.Q. Xie, S. Cheng, Y. Yang, J. Yan, H. Zhang, W.M. Chai, B. Schmidt, F.H. Yan. DualEnergy CT for Patients Suspected of Having Liver Iron Overload: Can Virtual Iron Content Imaging 
Accurately Quantify Liver Iron Content? Radiology, 277, 95 (2015).

8. S. Werner, B. Krauss, U. Haberland, M. Bongers, U. Starke, T. Bakchoul. Dual-energy CT for liver iron quantification in patients with haematological disorders.Eur Radiol. 2018, Nov 7, [Epub ahead of print].
9. P.J. Pickhardt, P.M. Graffy, S.B. Reeder, D. Hernando, K. Li. Quantification of Liver Fat Content With Unenhanced MDCT: Phantom and Clinical Correlation With MRI Proton Density Fat Fraction. AJR Am J Roentgenol, 211, 151 (2018). 